This is the final peer-reviewed accepted manuscript of:

3 Observational Study on Cryptosporidiosis in an Equine Perinatology Unit

Aliai Lancia*, Jole Mariella ${ }^{a}$, Eleonora lacono ${ }^{a}$, Monica Caffara $^{a}$, Silvia Piva ${ }^{a}$, Roberta Galuppi ${ }^{a}$, Carolina Castagnetti ${ }^{\mathrm{a}}$

The final published version is available online at: https://doi.org/10.1016/j.jevs.2018.09.009

8

9

10

11 This manuscript version is made available under the Creative Commons Attribution-

12 NonCommercial-NoDerivs (CC BY-NC-ND) 4.0 International License

13 (http://creativecommons.org/licenses/by-nc-nd/4.0/)

14 


\section{Observational Study on Cryptosporidiosis in an Equine Perinatology Unit}

17 Aliai Lanci ${ }^{\mathrm{a} *}$, Jole Mariella ${ }^{\mathrm{a}}$, Eleonora Iacono ${ }^{\mathrm{a}}$, Monica Caffara ${ }^{\mathrm{a}}$, Silvia Piva ${ }^{\mathrm{a}}$, Roberta Galuppi ${ }^{\mathrm{a}}$,

18 Carolina Castagnetti ${ }^{\mathrm{a}}$

19

20 *Corresponding author: Aliai Lanci, aliai.lanci2@unibo.it

21 The work was carried out at: Department of Veterinary Medical Sciences, University of Bologna, Via

22 Tolara di Sopra 50, 40064, Ozzano dell’Emilia (Bologna), Italy

$23{ }^{a}$ Affiliation: Department of Veterinary Medical Sciences, University of Bologna, Via Tolara di Sopra

2450,40064 , Ozzano dell'Emilia (Bologna), Italy

25 
The present study aimed to describe clinical signs of cryptosporidiosis in neonatal foals hospitalized in an Equine Perinatology Unit (EPU) and to compare the clinical signs between Cryptosporidium parvum and Cryptosporidium horse genotype infection. The study was divided into 2 parts. In the retrospective study, 9 foals infected by $C$. parvum were considered. In the prospective study, 70 foals, less than 15 days old, were prospectively included. Historical and clinical data were recorded and, in the prospective study, multiple fecal samples were collected. Cryptosporidium parvum $(\mathrm{n}=13)$ and Cryptosporidium horse genotype $(\mathrm{n}=7)$ were isolated. In 4 foals, there was a mixed infection with both the Cryptosporidium. Diarrhea, when present, showed similar duration and characteristics. Sixteen foals showed decreased abdominal sounds and colic pain before evidence of diarrhea. Nineteen foals had hyperthermia at least once. Although survival rates were similar between $C$. parvum (77\%), C. horse genotype (100\%) and cryptosporidial mixed infection (100\%), foals affected by $C$. parvum presented anorexia $(\mathrm{P}<0.0031)$ and received specific therapy $(\mathrm{P}<0.014)$ more frequently than the others. Recorded data strengthen the think that $C$. parvum infection is more severe in foals, suggesting that they would have developed host adaptations in response to the $C$. horse genotype or that $C$. parvum is a more pathogenic strain. Since healthy and asymptomatic foals can shed oocysts of Cryptosporidium spp., students and staff should always wear the personal protective equipment to avoid zoonotic infection.

Keywords: foal, Cryptosporidium, diarrhea, zoonosis. 


\section{INTRODUCTION}

Diarrhea is a very common clinical sign in newborn foals and could be a sign of infectious diseases, hypoxic gut injury, nutritional upsets or changes in intestinal flora, that quickly give rise to systemic manifestations [1,2]. The most common causes of diarrhea in newborn foals are: Rotavirus, Clostridium perfringens and difficile, Salmonella spp and Cryptosporidium [3].

Among parasites, Cryptosporidium was described for the first time in the 1978 by Snyder et al. [4] in 5 immunodeficient Arabian foals with diarrhea. The authors thought that this parasite only affected immunocompromised foals, but subsequently it has been associated with sporadic and/or outbreak of diarrhea even in immunocompetent horse [5,6].

Because of the high economic losses, due to growth retardation determined by diarrhea, much more has been written about cryptosporidiosis in calves, lambs and kids [7,8]. In these animals, cryptosporidiosis, mainly due to Cryptosporidium parvum, has been described as severe disease, characterized by yellow, malodorous feces, with consistency from soft to liquid, associated with depression, abdominal pain and anorexia [8-10].

In horse, until 2003 only Cryptosporidium parvum was known [11-14]. In 2003, Cryptosporidium horse genotype was described for the first time in Przewalski adult horse [15], and subsequently also isolated in healthy foals, less than one month old, in the New York State [16].

Epidemiological studies about cryptosporidial infection in horses were conducted in Louisiana [17], United Kingdom [18], New Zealand [13], New York State [16], Trinidad [19], Sao Paulo State [20], Algeria [21], Kentucky [22], Belgium, The Netherlands, Germany and Greece [23], China [24] and Brazil [25]. In particular, studies conducted in different farms of Central Italy revealed a great variability of prevalence, from $0 \%$ to $31.25 \%[6,14,26]$.

Many papers reported the prevalence of Cryptosporidium spp. in faeces of healthy adult horses and foals [12,16, 20,21,23,27,28], but clinical signs and risk factors are still unclear [14,29].

In the framework of a project about cryptosporidiosis in foals hospitalized at the Equine Perinatology Unit "Stefano Belluzzi" (EPU), of the Department of Veterinary Medical Sciences, Alma Mater 
Studiorum - University of Bologna, where Cryptosporidium parvum e Cryptosporidium horse genotype were isolated [26,30,31], the present study aimed to describe the clinical signs of cryptosporidiosis.

\section{MATERIALS AND METHODS}

\section{Experimental design}

The present study was divided into 2 parts:

Retrospective study - 6 foals (5 Italian Trotter and 1 Saddlebred) from foaling season 2007 and 3 foals (2 Italian Trotter and 1 Quarter horse) from foaling season 2011 were included. All these animals, hospitalized at the EPU, showed diarrhea and a fecal sample positive for Cryptosporidium parvum by both modified Ziehl-Neelsen [32] and PCR [26].

Prospective study (foaling seasons 2012 and 2013) - all foals, less than 15 days old, hospitalized at EPU during the foaling seasons 2012 and 2013 with or without diarrhea, were prospectively included.

\section{Data collection}

All the foals were subjected to a complete clinical examination at admission, and the following data were recorded: breed, sex, gestational age (days), age at admission (hours), blood culture result, serum IgG concentration (mg/dL), neutrophil blood count, and diagnosis. They were classified on the basis of the most life-threatening condition at admission following the guidelines reported by Castagnetti et al. (2010) [33]. Failure of passive transfer of immunity (FPT) was defined when, at more than 18 hours of life, serum IgG concentration was $<800 \mathrm{mg} / \mathrm{dL}$. During the hospitalization, foals were clinically evaluated at least twice a day and the following data were recorded: rectal temperature, age and days of hospitalization at the onset of symptoms, gastrointestinal symptoms such as anorexia, diarrhea (presence, type and duration), colic pain, characteristics of abdominal sounds (normal, increased, decreased, or absent), necessity of treatment due to cryptosporidiosis and type of treatment 
(adsorbents, lactic ferments, antibiotics, antidiarrheal drugs, analgesic drugs, intravenous fluids and plasma, total parenteral nutrition - TPN), hospitalization length and outcome. Finally, whether foals with cryptosporidiosis survived, died spontaneously, or were euthanized was recorded.

Foals with diarrhea were isolated inside the unit, applying a specific internal isolation protocol. All the operators were trained to apply the correct isolation practices with infectious animals (personal protective equipment - PPE: disposables gloves, boot covers and plastic smocks) and the boxes were always cleaned with specific and dedicated shovel, broom and barrow.

\section{Stool sample collection}

During the foaling seasons 2007 and 2011, fecal samples were collected only in case of diarrhea, while during the foaling seasons 2012 and 2013, the protocol reported by Galuppi et al. (2015) [26] was applied as follow: in foals born at EPU, fecal samples were collected 4 days after birth and then every 2 days until discharge; in foals hospitalized after birth (with or without diarrhea), fecal samples were collected at admission, 4 days later and then every 2 days until discharge. In all animals, sampling was made directly from rectal ampulla to avoid environmental contamination. Immediately after collection, each sample was identified through animal's name, date, progressive number and it was kept refrigerated $\left(+4^{\circ} \mathrm{C}\right)$ until processing. If the foal had not expelled meconium at admission, sampling was performed at the onset of the first milk feces.

All the fecal samples were subjected to bacteriological culture (E. coli, Clostridium spp, Salmonella spp), Rotavirus and Cryptosporidium analysis.

Each fecal sample was subjected to microscopic and molecular analysis for Cryptosporidium spp. as previously described $[26,30]$. Each stool specimen was homogenized in distilled water, filtered through gauze and centrifuged at $900 \times \mathrm{g}$ for $30 \mathrm{~min}$, the sediment was in part streaked onto a slide, stained with modified Ziehl-Neelsen (Henriksen and Pohlens, 1981) method and observed under a light microscope $\left(400 \times\right.$ and $1000 \times$ magnification), in part frozen at $-20{ }^{\circ} \mathrm{C}$ for molecular analysis. The 
rRNA. The positive samples were genotyped by restriction fragment length polymorphism (RFLP) analysis and subtyped by nested PCR of the $60 \mathrm{kDa}$ glycoprotein (gp60) gene following the conditions of [34].

\section{Statistical analysis}

Data were analyzed for normality with the Kolmogorov-Smirnov test. Given the non-normal distribution of data, nonparametric tests were used for statistical analysis.

Fisher's exact test was used to test the differences between the categorical variables. The differences in proportion between diarrheic foals positive to Cryptosporidium spp and negative diarrhoeic foals were tested for: sex, age (greater or less than 72 hours of life), anorexia, prematurity, perinatal asphyxia syndrome, FPT, sepsis and exitus, The differences between foals affected by two genotypes of Cryptosporidium were tested for: sex, anorexia, necessity of treatment, characteristics of abdominal sounds, hypertermia, FPT and exitus. Kruskal -Wallis test and Student $\mathrm{t}$ test were performed to determine the differences between numeric variables (age at admission, gestational age, neutrophil count, duration of diarrhea and hospitalization length) between foals with and without diarrhea and between foals affected by two genotypes of Cryptosporidium. Summary data were reported as median \pm standard error $(\mathrm{SE})$. All analyses were performed with a commercial software (Analyse-it Software Ltd., Leeds, West Yorkshire, England). Values of $\mathrm{P}<0.05$ were considered significant.

\section{RESULTS}

Retrospective study

In the 2007 foaling season, the onset of clinical signs of cryptosporidiosis in the 6 included foals occurred at $6 \pm 1.6$ days of life (range 1.5-14) and $5.7 \pm 1.7$ days of hospitalization (range $0-14 ; 3$ foals were born at EPU). None foal died or was euthanized and the median hospitalization length was 35 \pm 5.1 days (range 17-45). 
During the 2011 foaling season, none of the three enrolled foals was born at EPU. The onset of clinical signs of cryptosporidiosis occurred at $9 \pm 1.9$ days of life (range 5-13) and $7 \pm 2.1$ days of hospitalization (range 3.5-12.5). One foal (1/3 foals, 33.3\%) was discharged after 40 days of hospitalization. One foal (1/3 foals, 33.3\%) was euthanized with the owner's consent after 27 days due to financial constraints and one foal (1/3 foals, 33.3\%) after 20 days due to worsening health conditions.

Clinical data collected at admission (sex, age at admission, gestational age, FPT, neutrophil count and major diagnosis) are shown in Table 1 while symptoms and outcome are listed in Table 2.

\section{Prospective study}

During the 2012 and 2013 foaling seasons, 70 foals were hospitalized at EPU. Twelve/70 foals were excluded from the statistical analysis: in 11/12 (91.7\%) foals the sampling protocol was not complete, since they were euthanized or died within 24 hours of hospitalization without showing clinical signs of Cryptosporidiosis; $1 / 11(9.1 \%)$ foal was excluded because affected by a mixed infection (Rotavirus and both Cryptosporidium genotypes). Therefore, 58 foals were considered in the study (22 Italian Trotters, 19 Saddlebreds, 9 Arabian Horses, 5 Quarter Horses, 1 Paint Horse, 1 Missouri Fox Trotter and 1 pony Shetland).

Eighteen/58 (1.1\%) foals were born at EPU and 40/58 (68.9\%) were referred. Seven foals were euthanized for poor clinical condition: $1 / 7(14.3 \%)$ foal was positive to $C$. parvum, while none of the euthanized foals was positive for Cryptosporidium spp.

Clinical data collected at admission are shown in Table 1; symptoms and outcome are listed in Table 2.

The onset of clinical signs in positive foals started at $5 \pm 0.9$ days of life (range 1-13), after $2.5 \pm 0.7$ days of hospitalization (range 0-6.5). Fifteen out of 58 animals (25.8\%) tested positive for Cryptosporidium spp.: 4/15 (6.9\%) for C. parvum (2 in 2012 and 2 in 2013), 7/15 (12\%) for Cryptosporidium horse genotype (in 2012) and 4/15 (6.9\%) had mixed infection with both 
Cryptosporidium spp (in 2012). In foals positive for C. parvum, the duration of hypomotility was 1 \pm 0.1 days (range $0.5-2$ ). In foals with cryptosporidial mixed infection, the duration of hypomotility was $1.5 \pm 0.1$ days (range 1-1.5).

No significant differences were found in clinical data collected at admission between foals negative and positive for Cryptosporidium spp. (P >0.05), using Student's t test and Fisher's Exact test.

All the 24 positive foals showed gastrointestinal symptoms, except one completely asymptomatic foal (4.2\%) positive for Criptosporidium horse genotype. Diarrhea, when present, showed similar characteristics in both Criptosporidium infections: watery, yellowish and foul smelling. In 16/24 (66.7\%) foals, dehydration, anorexia and loss of electrolyte lead to the necessity of intensive therapy with intravenous fluids, plasma and TPN. During diarrhea episodes, all the foals showed increased abdominal sounds. In 16/24 (66.7\%) foals, before evidence of diarrhea, the auscultation of the abdomen revealed decreased abdominal sounds. Colic pain symptoms as rolling, bruxism, abrade or get in decubitus continuously was observed in 16/24 (66.7\%) foals. In 11/16 (68.7\%) all the mentioned above symptoms were present.

Although survival rates were not significantly different between the three groups (C. parvum 77\%, Cryptosporidium horse genotype 100\% and cryptosporidial mixed infection 100\%), Fisher's exact test revealed that foals affected by $C$. parvum have more probability to present anorexia $(\mathrm{P}<0.0031)$ and to receive specific treatment $(\mathrm{P}<0.014)$ than the others.

\section{DISCUSSION}

To the authors' knowledge, this is the first study on cryptosporidiosis symptomatology in hospitalized neonatal foals caused by $C$. parvum and Cryptosporidium horse genotype.

There are few clinical studies about cryptosporidiosis in hospitalized foals; two of them describe cryptosporidial infection in immunodeficient Arabian foals $[4,11]$ and both demonstrated that foals with Severe Combined Immunodeficiency (SCID) developed severe diarrhea and persistent cryptosporidial infection following experimental challenge with C. parvum oocysts [11]. 
In the prospective study, the prevalence of Cryposporidium spp. in hospitalized foals was $11.2 \%$, but this finding cannot be compared with other epidemiological surveys performed on farms in healthy foals and adult horses. In the authors' opinion, an equine intensive care unit could be considered a high-risk setting due both to the presence of sick and immunocompromised foals and to the high environmental resistance of the oocysts which are not inactivated by the most common disinfectants and can persist for a long period of time.

There are only two case reports about cryptosporidial infection in foals: one in a 9 days old Arabian colt infected by C. parvum bovine genotype [35] and another in an Arabian foal with SCID [36]. In the 2003, Grinberg et al. [5] reported a severe outbreak of foal diarrhea, caused by $C$. parvum cattle genotype, in a thoroughbred farm in New Zealand that lasted for one month and, during that period, nine foals suffered from acute, mild to severe disease, accompanied by dehydration and weakness. 
PAS had C. parvum diarrhea lasting 23 days and, due to the poor clinical condition, it was euthanized, while in a case reported by Imhasly et al. (2009) [35], the colt was discharged in good clinical conditions after 10 days.

The most important findings emerging from the present study is the presence of decreased bowel sounds before appearance of diarrhea and the anorexia. Physical evidence of diarrhea may not be seen early in the course of the disease, whether it is mild or severe [2]. Two mechanisms are implicated in the pathogenesis of intestinal ileus: inflammation and distention. Local inflammation is probably due to the local overproduction of nitric oxide acid caused by the upregulation of inducible nitric oxide synthase by resident macrophages. Nitric oxide is the inhibitory neurotransmitter of the nonadrenergic, noncholinergic system [39,40]. Excessive distention results in inhibition of motility within the distended segment of bowel $[41,42]$. In the authors' opinion, this phase of hypomotility can mislead the clinician, who does not promptly recognize the incoming diarrhea and does not isolate the foal. In this way, the infected foal may become a source of infection for the other hospitalized foals and for the personnel. Also Xiao and Herd (1994) [43] suggested that the main source of Cryptosporidium spp. infection in foal was infected foals, while mares were the major source of Giardia infection. In fact, in a more recent study of Galuppi et al. (2015) [26], no mare was positive to Cryptosporidium spp. This finding suggests the importance of confining hospitalized foals with hypomotility and abdominal discomfort in isolation until the presumptive diagnosis. Anorexia was significantly prevalent in foals affected by $C$. parvum. In fact, no one foal with Cryptosporidium horse genotype and mixed infection stopped to nurse from the mare.

Previously, Cryptosporidium horse genotype was identified twice without clinical symptomatology, one in a Prezewalski's wild horse foal in Prague Zoo [15], and in nine foals 1-10 weeks of age in 250 New York [16].

Restriction of oral intake of milk can be very useful in the treatment of neonatal or young foals with diarrhea, since it can be exacerbated by the osmotic influences of milk intake. 'Resting' of the gastrointestinal tract to allow for recovery of damaged enterocytes can be a beneficial part of therapy. 
254 Neonates must be deprived of milk intake cautiously, as glucose energy stores are limited at this age.

255 Parenteral nutrition may be necessary and it allows increased periods of time without milk intake [2].

256 In the present study, the necessity of treatment of the cryptosporidial diarrhea was significantly 257 prevalent in foals affected by C. parvum. Moreover all the 7 foals infected only by Cryptosporidium 258 horse genotype and the 4 foals with mixed infection were discharged, while, among C. parvum 259 infected foals, 3 were euthanized due to the poor clinical condition and another one died soon after 260 the admission. Also Grinberg et al. (2003) [5] reported that, during a severe outbreak of C. parvum 261 diarrhea, two foals died from the disease and a third was euthanized due to a severe condition despite 262 intensive treatments.

263 These results could suggest that $C$. parvum infection is more severe in foals, probably because they 264 may have developed host adaptations in response to the Cryptosporidium horse genotype or because 265 C. parvum is a more pathogenic strain.

266 In human and bovine, the species most affected, several specific treatments are suggested for 267 cryptosporidiosis, but their specificity and efficacy are not completely verified [44-45]. In this 268 population, all foals affected by $C$. parvum and the $50 \%$ of foals with mixed infection needed an 269 intensive supportive therapy, while this has been necessary only for the $28.6 \%$ of foals affected by 270 Cryptosporidium horse genotype.

271 In healthy foals, a frequent cause of diarrhea is attributed to the mare's post-foaling oestrus. "Foal 272 heat diarrhea" affects up to $80 \%$ of foals 5-15 days-old, which remain bright and alert, continue to 273 nurse, but produce soft to watery faeces; this diarrhea is usually self-limiting and rarely requires 274 treatment [46-48]. Viruses, bacteria or parasites are suspected of predisposing foals to "foal heat 275 diarrhea" or to represent a complication [6,46]. It might be supposed that Cryptosporidium horse 276 genotype could be the parasite that causes the foal heat diarrhea. However, cases of neonatal 277 cryptosporidiosis in foals might remain undiagnosed and managed as generic "foal heat diarrhea" $278[6,13]$, supporting the widespread of the infection. 
In conclusion, the appropriate identification of potentially infected animal provides the basis to prevent the widespread of infection to other foals, especially if hospitalized in intensive therapy.

Since healthy and asymptomatic foals can shed oocysts of Cryptosporidium horse genotype, students and staff should always wear the personal protective equipment to avoid zoonotic infection.

\section{Acknowledgements}

This study was funded by "Progetti competitivi per Ricercatori" grant of Department of Veterinary Medical Sciences (DIMEVET), Alma Mater Studiorum - University of Bologna, Budget integrato 2011.

\section{REFERENCES}

[1] Barr, B., 2007: Neonatal foal diarrhea. The North American Veterinary Conference. 82-83.

[2] Bernard WV. 2012: Alimentary tract disorders. In: Equine Paedriatic medicine. Bernard, W.V., Barr, B., Manson Publishing, London.

[3] Oliver-Espinosa, O., 2018: Foal Diarrhea: Established and Postulated Causes, Prevention, Diagnostics, and Treatments. Vet Clin North Am Equine Pract.34, 55-68.

[4] Snyder, S.P., England, J.J., and A.E. McChesney 1978: Cryptosporidiosis inimmunodeficient Arabian foals. Vet Pathol 15, 12-17.

[5] Grinberg, A., Oliver, L., Learmonth, J.J., Leyland, M., Roe, W., and W.E. Pomroy 2003: Identification of Cryptosporidium parvum 'cattle' genotype from a severe outbreak of neonatal foal diarrhoea. Vet Rec 153, 628-631.

[6] Perrucci, S., Buggiani, C., Sgorbini, M., Cerchiai, I., Otranto, D., and D. Traversa 2011: Cryptosporidium parvum infection in a mare and her foal with foal heat diarrhoea. Vet Parasitol 182, 333-336.

[7] Sanford, S.A., and G.K.A. Josephson 1982: Bovine cryptosporidiosis: clinical and pathological findings in forty-two scouring neonatal calves. Can Vet J 23, 340-343. 
[8] De Graaf, D.C., Vanopdenbosch, E., Ortega-Mora, L.M., Abbassi, H., and J.E. Peeters 1999: A review of the importance of cryptosporidiosis in farm animals. Int J Parasitol 29, 1269-1287.

[9] Ortega-Mora, L.M., and S.E. Wright 1994: Age-related resistance in ovine cryptosporidiosis: patterns of infection and humoral immune response. Infect Immun 62, 5003-5009.

[10] Koudela, B., and V. Jiri 1997: Experimental cryptosporidiosis in kids. Vet Parasitol 71, 273281.

[11]Bjorneby, J.M., Leach, D.R., and L.E. Perryman 1991: Persistent cryptosporidiosis in horses with severe combined immunodeficiency. Infect Immun 59, 3823-3826.

[12] Majewska A.C., Solarczyk P., Tamang L., T.K. Graczyk 2004: Equine Cryptosporidium parvum infections in western Poland. Parasitol Res 93, 274-278.

[13] Grinberg, A., Pomroy, W.E., Carlslake, H.B., Shi, Y., Gibson, I.R., and B.M. Drayton 2009. A study of neonatal cryptosporidiosis of foals in New Zealand. New Zeal Vet J 57, 284-289.

[14] Veronesi, F., Passamonti, F., Cacciò, S., Diaferia, M., Piergili, and D. Fioretti 2010: Epidemiological survey on equine Cryptosporidium and Giardia infections in Italy and molecular characterization of isolates. Zoonoses and Public Health 57, 510-517.

[15] Ryan, U., Xiao, L., Read, C., Zhou, L., Lal, A.A., and I. Pavlasek 2003: Identificationof novel Cryptosporidium genotypes from the Czech Republic. Appl Environ Microbiol 69, $4302-4307$.

[16]Burton, A.J., Nydam, D.V., Dearen, T.K., Mitchell, K., Bowman, D.D., and L. Xiao 2010: The prevalence of Cryptosporidium, and identification of the Cryptosporidium horse genotype in foals in New York State. Vet Parasitol 174, 139-144.

[17] Coleman, S.U., Klei, T.R., French, D.D., Chapman, M.R., and R.E. Corstvet 1989: Prevalence of Cryptosporidium sp. in equids in Louisiana. Am J Vet Res 50, 575-577.

[18]Netherwood, T., Wood, J.L., Townsend, H.G., Mumford, J.A., and N. Chanter 1996: Foal diarrhoea between 1991 and 1994 in the United Kingdom associated with Clostridium 
perfringens, rotavirus, Strongyloides westeri and Cryptosporidium spp. Epidemiol infect 117, 375-383.

[19]Harris, R., Sankar, K., Small, J.A., Suepaul, R., Stewart-Johnson, A., and A. Adesiyun 2012: Prevalence and characteristics of enteric pathogens detected in diarrhoeic and non-diarrhoeic foals in trinidad. Veterinary medicine international, 1-9.

[20]Inácio, S.V., de Brito, R.L., Zucatto, A.S., Coelho, W.M., de Aquino, M.C.,Aguirre Ade, A., Perri, S.H., Meireles, M.V., and K.D. Bresciani, 2012: Cryptosporidium spp. infection in mares and foals of the north-west region of São Paulo State, Brazil. Rev Braz Parasitol Vet $21,355-358$.

[21]Laatamna, A.E., Wagnerová, P., Sak, B., Květoňová, D., Aissi, M., Rost, M., and M.Kváč, 2013: Equine cryptosporidial infection associated with Cryptosporidium hedgehog genotype in Algeria. Vet Parasitol 197, 350-353.

[22] Slovis, N.M., Elam, J., Estrada, M., and C.M. Leutenegger 2014: Infectious agents associated with diarrhoea in neonatal foals in central Kentucky: a comprehensive molecular study. Equine Vet J 46, 311-316.

[23] Kostopoulou, D., Casaert, S., Tzanidakis, N., van Doorn, D., Demeler, J., von SamsonHimmelstjerna, G., Saratsis, A., Voutzorakis, N., Ehsan, A., Doornaert, T., Looijen, M., De Wilde, N., Sotiraki, S., Claerebout, E. and T.Geurden 2015: The occurrence and genetic characteriation of Cryptosporidium and Giardia species in foals in Belgium, The Netherlands, Germany and Greece. Vet Parasitol 211, 170-174.

[24]Liu, A., Zhang, J., Zhao, J., Zhao, W., Wang, R., and L., Zhang 2015: The first report of Cryptosporidium andersoni in horses with diarrhea and multilocus subtype analysis. Parasit Vectors 8, 483.

[25] Toscan, G., Pereira, R.C.F., Vogel, F.S.F., and L.A., Sangioni 2010: Cryptosporidium spp. in traction horses in Santa Maria, RS, Brazil. Arq Bras Med Vet Zootec 62, 211-213. 
[26] Galuppi, R., Piva, S., Castagnetti, C., Iacono, E., Tanel, S., Pallaver, F., Fioravanti, M.L., Zanoni, R.G., Tampieri, M.P., and M. Caffara 2015: Epidemiological survey on Cryptosporidium in an Equine Perinatology Unit. Vet Parasitol 210, 10-18.

[27] Johnson, E., Atwill, E.R., Filkins, M.E., and J. Kalush 1997: The prevalence of shedding of Cryptosporidium and Giardia spp. based on a single fecal sample collection from each of 91 horses used for backcountry recreation. J Vet Diagn Invest 9, 56-60.

[28]De Souza, P.N.B., Bomfim, T.C.B., Huber, F., Abboud, L.C.S., and R.S. Gomes 2009: Natural infection by Cryptosporidium sp., Giardia sp. and Eimeria leuckarti in three groups of equines with different handlings in Rio de Janeiro, Brazil. Vet Parasitol 160, 327-333.

[29] Mallicote, M., House, A.M., and L.C. Sanchez, 2012: A review of foal diarrhoea from birth to weaning. Equine Vet Educ 24, 206-214.

[30]Caffara, M., Piva, S., Pallaver, F., Iacono, E., Zanoni, R.G., and R. Galuppi 2013: Molecular characterization of Cryptosporidium spp. from foals in Italy. Vet J 198, 531-33.

[31]Piva, S., Caffara, M., Pasquali, F., Castagnetti, C., Iacono, E., Massella, E., Zanoni, R.G., and R. Galuppi 2016: Detection and quantification of Cryptosporidium oocysts in environmental surfaces of an Equine Perinatology Unit. Prev Vet Med 131, 67-74.

[32] Henriksen, S.A., Pohlens, J.F., 1981: Staining of cryptosporidia by a modi-fied Ziehl-Neelsen technique. Acta Vet Scand 22, 628-631.

[33] Castagnetti, C., Pirrone, A., Mariella, J., and G. Mari 2010: Venous blood lactate evaluation in equine neonatal intensive care. Theriogenology $73,343-57$.

[34]Xiao, L., Ryan, U.M., 2008. Molecular epidemiology. Cryptosporidium and Cryptosporidiosis. CRC Press and IWA Publishing, Boca Raton (USA), 119-171.

[35]Imhasly, A., Frey, C.F., Mathis, A., Straub, R., and V. Gerber, 2009: Cryptosporidiosis (C. parvum) in a foal with diarrhea. Schweiz Arch Tier 151, 21-26. 
[36] Mair, T.S., Taylor, F.G., Harbour, D.A., and G.R. Pearson 1990: Concurrent cryptosporidium and coronavirus infections in an Arabian foal with combined immunodeficiency syndrome. Vet Rec126, 127-130.

[37]McGuire, T.C., Crawford, T.B., Hallowell, A.L., and L.E. Macomber 1977: Failure of colostral immunoglobulin transfer as an explanation for most infections and deaths of neonatal foals. J Am Vet Med Assoc170, 1302-1304.

[38]Perkins, G.A., and B. Wagner 2015: The development of equine immunity: Current knowledge on immunology in the young horse. Equine Vet J 47, 267-274.

[39]Rakestraw, P.C., Snyder, J.R., Woliner, M.J., Sanders, K.M., and C.W. Shuttleworth 1996: Involvement of nitric oxide in inhibitory neuromuscular transmission in equine jejunum. Am J Vet Res 57, 1206-1213.

[40] Malone, E.D., Kannan, M.S., Brown, D.R., Turner, T.A., and A.M. Trent 1999: Adrenergic, cholinergic, and nonadrenergic-noncholinergic intrinsic innervation of the jejunum in horses. Am J Vet Res 60, 898-904.

[41]MacHarg, M.A., Adams, S.B., Lamar, C.H., and J.L. Becht 1986: Electromyographic, myomechanical, and intraluminal pressure changes associated with acute extraluminal obstruction of the jejunum in conscious ponies. Am J Vet Res 47, 7-11.

[42]Lester, G.D. 2004: Gastrointestinal Ileus in Equine Internal Medicine. Reed, S.M., Bayly, W.M., Sellon, D.C., 2004. Elsevier, USA, 815-820.

[43] Xiao, L., and R.P. Herd 1994: Epidemiology of equine Cryptosporidium and Giardia infections. Equine Vet J 26, 14-17.

[44] Meganck, V., Hoflack, G., Piepers, S., and G. Opsomer 2015: Evaluation of a protocol to reduce the incidence of neonatal calf diarrhoea on dairy herds. Prev Vet Med 118, 64-70.

[45] Sparks, H., Nair, G., Castellanos-Gonzalez, A., and A.C. Jr White 2015: Treatment of Cryptosporidium: What We Know, Gaps, and the Way Forward. Curr Trop Med Rep 2, 181187. 
[46] Masri, M.D., Merritt, A.M., Gronwall, R., and C.F. Burrows 1986: Fecal composition in foal heat diarrhea. Equine Vet J 18, 301-306.

406

[47]Freestone, J., 2002: Foal heat diarrhea. In: Mair, T., Divers, T., Ducharme, N., Manual of Equine Gastroenterology, pp 493. WB Saunders, Philadelphia, USA.

[48] McAuliffe, S.B., and N.M. Slovis 2008: Colour Atlas of Diseases and Disorders of the Foal. 409 Pp.103. Saunders Elsevier Ed, UK. 